\title{
Post-encoding stress enhances mnemonic discrimination of negative stimuli
}

\author{
Tony J. Cunningham, ${ }^{1,4}$ Stephanie L. Leal, ${ }^{2,4}$ Michael A. Yassa, ${ }^{3}$ and Jessica D. Payne ${ }^{1}$ \\ ${ }^{1}$ Department of Psychology, University of Notre Dame, Notre Dame, Indiana 46556, USA; ${ }^{2}$ Helen Wills Neuroscience Institute, \\ University of California, Berkeley, Berkeley, California 94720, USA; ${ }^{3}$ Department of Neurobiology and Behavior and Center for the \\ Neurobiology of Learning and Memory, University of California, Irvine, Irvine, California 92697, USA
}

\begin{abstract}
Stress influences how we remember emotional events and how these events shape future behaviors. However, the impact of stress on memory specificity for emotional events has yet to be examined. To this end, the present study utilized a mnemonic discrimination task that taxes hippocampal pattern separation, the process of distinguishing between overlapping experiences, thereby allowing us to better understand the mechanisms by which stress affects gist versus detail memory of emotional events. Participants encoded scenes composed of negative or neutral objects placed on neutral backgrounds and then underwent a psychosocial stressor or matched control task. Twenty-four hours later during testing, objects were presented separately, with some identical old objects (targets), some new objects (foils), and some similar but not identical objects (lures). Target recognition was enhanced for negative compared to neutral objects in both the stress and control groups. Interestingly, post-encoding stress selectively enhanced mnemonic discrimination of negative versus neutral objects, which was not the case in the control group. Measures of salivary cortisol revealed a quadratic inverted U relationship between negative mnemonic discrimination and cortisol increase. These findings suggest that moderate cortisol release following stress is associated with enhanced memory precision for negative information.
\end{abstract}

Many events in our lives tend to share key features. They take place in the same location, occur with the same people, and can involve the same emotions, yet we are able to maintain separate records of such experiences. Pattern separation, the process of disambiguating similar representations as distinct from one another, is one mechanism for reducing interference of overlapping experiences and is thought to be subserved by the hippocampus (Marr 1971; Treves and Rolls 1992; Shapiro and Olton 1994; McClelland et al. 1995; Yassa and Stark 2011). Mnemonic discrimination tasks, which assess the ability to discriminate highly similar stimuli in memory by calculating a lure discrimination index (LDI), have been used reliably in the past to examine pattern separation in humans (cf. Leal and Yassa 2018 for review). However, the impact of stress on individual facets of performance such as target recognition and lure discrimination has not been evaluated in detail.

Numerous studies suggest that stress hormones play a critical role in the formation and storage of emotional memories (Gold and McGaugh 1975; McGaugh 2004; McIntyre et al. 2012; Cunningham et al. 2014). These hormones boost activity in the basolateral amygdala, and enhance connectivity among the amygdala and other memory-relevant regions of the brain, including the hippocampus (McGaugh 2004; Roozendaal et al. 2009). The potentiation of this network by stress is thought to benefit the consolidation of emotionally salient memories (e.g., Buchanan and Lovallo 2001; Vyas et al. 2002; Cahill et al. 2003; Abercrombie et al. 2006; Payne et al. 2007; for reviews, see de Quervain et al. 2009; Wolf 2009), sometimes at the cost of neutral memories that likely have less adaptive value (Payne et al. 2006, 2007). For instance, preencoding stress exposure has been shown to enhance long-term memory for an emotionally arousing slide show, but impair memory for a matched neutral slide show (Payne et al. 2007). Similarly, participants who were exposed to stress after watching a slide show consisting of neutral and emotionally arousing slides remembered

\section{${ }^{4}$ These authors contributed equally to this work.} Corresponding author: jpayne7@nd.edu

Article is online at http://www.learnmem.org/cgi/doi/10.1101/lm.047498.118. more emotional versus neutral slides than nonstressed control participants (Cahill et al. 2003; Nielsen et al. 2013). In general, a large body of research suggests that stress exposure during the early consolidation window can enhance emotional memory (e.g., Cahill et al. 2003; Smeets et al. 2008a,b; Shields et al. 2017). However, the accuracy and level of detail with which these memories are stored remain equivocal. Tasks that tax hippocampal pattern separation may offer a unique perspective into this dynamic. Rather than testing overlapping gist and detail information such as in the emotionally arousing slide show discussed above, these tasks offer a lure discrimination measure that is based on the parametric manipulation of the similarity of the stimuli, thus taxing hippocampal pattern separation and may offer a mechanism underlying stress-related enhancements in emotional memory.

Thus far, one study has applied a mnemonic discrimination task to the memory of emotional stimuli, and found that target recognition (gist) was preserved for emotional versus neutral information, while lure discrimination (detail) was impaired for emotional versus neutral information (Leal et al. 2014a). This was then linked to increased hippocampal DG/CA3 and amygdala activity during accurate discrimination using high-resolution fMRI (Leal et al. 2014b) and suggests mnemonic discrimination tasks can inform us about the mechanisms underlying emotional memory consolidation. Further, individuals with depressive symptoms (e.g., a chronic stress syndrome that is often associated with elevations of the stress hormone cortisol, see Pariante and Miller 2001) show impaired lure discrimination for neutral items (Déry et al. 2013; Shelton and Kirwan 2013; Leal et al. 2014a). When performing a mnemonic discrimination task including negative stimuli, individuals with depressive symptoms showed enhanced lure

(C) 2018 Cunningham and Payne This article is distributed exclusively by Cold Spring Harbor Laboratory Press for the first 12 months after the full-issue publication date (see http://learnmem.cshlp.org/site/misc/terms.xhtml). After 12 months, it is available under a Creative Commons License (AttributionNonCommercial 4.0 International), as described at http://creativecommons. org/licenses/by-nc/4.0/. 
discrimination only for negative information (Leal et al. 2014a). This was not the case for target recognition. This suggests that lure discrimination measures may be more sensitive to memory processing in depressed individuals, which may, in part, be due to elevated stress and stress hormone release observed in depression (e.g., Yehuda et al. 1996; Abercrombie 2009), potentially leading to the enhanced amygdala activation in response to negative stimuli, also typically found in individuals with depression (Siegle et al. 2002, 2007; Roberson-Nay et al. 2006).

Determining the influence of acute stress on mnemonic discrimination of emotional information is an important next step in understanding how stress affects the veridicality of emotional memories. This question is critical in determining whether stress enhances the precise, detailed long-term storage of emotional information or if it promotes a more generalized, gist-based memory storage. Here we investigated the effect of post-encoding stress on memory for emotional and neutral stimuli. While stress often benefits the consolidation of emotionally arousing information (e.g., for review, see Payne and Nadel 2004; Payne et al. 2007; Wolf 2009), few studies have explored how stress affects the specificity of memory using tasks that rely on hippocampal pattern separation. We have included two measures of memory in this study to investigate more general memory effects for repeated items (i.e., target recognition) as well as a more specific memory measure of the ability to discriminate highly similar items (i.e., lure discrimination). We hypothesized that post-encoding stress (via the Trier Social Stress Test [TSST]) would lead to an enhancement in lure discrimination of negative, but not neutral information, not only because we found this effect in individuals with depressive symptoms previously, but also given that cortisol release is associated with enhanced amygdala-hippocampal connectivity and better subsequent memory for negative emotional information (e.g., van Stegeren et al. 2007; Vaisvaser et al. 2013). Additionally, we expected lure discrimination to be more sensitive than target recognition in identifying stress-related differences. Furthermore, similar to other studies in which the encoding period precedes the stressor (Cahill et al. 2003), we did not expect any effect of stress on neutral information.

\section{Results}

Participants arrived in the lab mid-afternoon and completed an incidental encoding task, rating 64 complex scenes (32 with a negative central object and 32 with a neutral central object, all placed on neutral backgrounds; Kensinger et al. 2006), before being randomly assigned to either a stress or control condition. After encoding, stress participants completed a psychosocial stressor (TSST, Kirschbaum et al. 1993) and control participants completed a matched control task. Saliva samples and self-report measures were collected for later cortisol/stress assessment. The next day, participants returned to the lab to perform an unexpected, self-paced recognition task, in which the scene objects and backgrounds were presented individually and one at a time. The recognition task included scene components that were identical to the objects and backgrounds that had been encoded ("same"), similar (but not identical) versions of the object or background, ("similar"), and new objects and backgrounds that had not been previously viewed ("new").

\section{Stressor efficacy}

\section{Physiological response}

To assess the impact of the TSST versus control manipulation on cortisol responsivity, we performed a 2 (Group: Stress vs. Control) $\times 6$ (Time: t0, t1, t2, t3, t4, t5) mixed ANOVA, with time as the within-subject factor and group as the between-subject fac- tor. This analysis revealed a significant Time $\times$ Group interaction $\left(F_{(5,44)}=3.2, P=0.009\right.$; partial $\eta^{2}=0.06$, observed power $\left.=0.88\right)$. Because participants' peak cortisol response to stressors can occur at different time points following stress exposure (Kudielka and Kirschbaum 2005; Otte et al. 2005; Kajantie and Phillips 2006; Kudielka et al. 2007), we subtracted baseline cortisol concentrations ( $\mathrm{t} 0$ ) from the maximum cortisol increase following the stressor ( $\mathrm{t} 1, \mathrm{t} 2, \mathrm{t} 3, \mathrm{t} 4$, or $\mathrm{t} 5$; note that this was at $\mathrm{t} 2$ for most participants [40\%]). Using this change score, there was a clear increase in cortisol concentrations in participants exposed to the stressor, compared to the control condition $\left(t_{(48)}=3.8, P<0.0001\right.$; see Fig. 1$)$. Additionally, individual group analyses comparing baseline cortisol concentrations to the maximum concentration following the stress manipulation revealed that the stress group had a significant increase in cortisol $\left(t_{(27)}=5.4, P<0.001\right)$, while the control group had no change in cortisol concentration from baseline $\left(t_{(21)}=0.2\right.$, $P=0.83)$. These findings confirm that the stress task successfully elicited a physiological stress response leading to increased cortisol concentrations.

\section{Subjective response}

To determine the impact of the TSST on the subjective experience of stress, the state-trait anxiety questionnaire (STAI) was given both during the initial acclimation period upon entering the lab and immediately following the stress manipulation. We conducted mixed ANOVAs, with time of assessment as the repeated measure, on ratings of state anxiety (STAI-state) in the stress and control groups. We found a main effect of group on reported anxiety $\left(F_{(1,48)}=\right.$ $12.9, P=0.001$, partial $\eta^{2}=0.21$, observed power $\left.=0.94\right)$ and an interaction between group and time of assessment $\left(F_{(1,48)}=17.2, P<\right.$ 0.001 , partial $\eta^{2}=0.26$, observed power $=0.98$ ). To determine the direction of these effects we used follow-up paired $t$-tests to examine the change in STAI-state score. Mirroring the cortisol data, participants in the stress group demonstrated a significant increase in reported anxiety from baseline to post-TSST $\left(t_{(27)}=5.7, P<0.001\right)$, while the control group showed no change in STAI-state score over time $\left(t_{(21)}=0.38, P=0.70\right)$. This finding confirms that, in addition to generating a significant increase in HPA axis activation, the stress task was also successful in elevating subjectively experienced stress.

\section{Stress enhances mnemonic discrimination of negative objects}

To determine the effect of stress on mnemonic discrimination of negative and neutral objects, we conducted a 2 (Emotion:

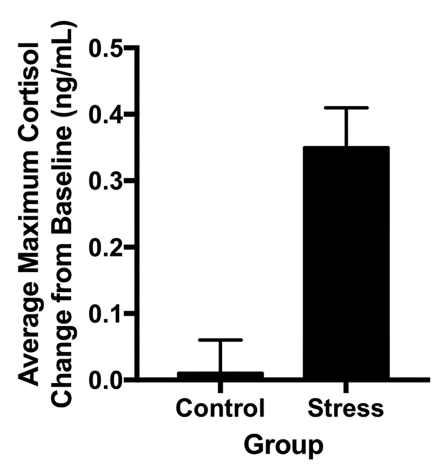

Figure 1. Average change in cortisol in Control and Stress Groups. The Stress group demonstrated a significant increase in salivary cortisol concentration from baseline to maximum increase during the 60-min period following the task compared to the control group $(P<0.0001)$. Error bars represent SEM. 
Negative vs. Neutral) $\times 2$ (Group: Stress vs. Control) ANOVA, with Emotion as a repeated factor on the calculated LDI measure for each valence (see Memory Analysis for how this was computed). Although there was no main effect of either emotion or group, critically, we found a significant interaction between emotion and group $\left(F_{(1,48)}=5.0, P=0.03\right.$, partial $\eta^{2}=0.09$, observed power $=$ 0.59; Fig. 2). Post-hoc contrasts revealed that the interaction was driven by enhanced mnemonic discrimination of negative objects in the stress group compared to the control group $\left(t_{(48)}=-2.7, P=\right.$ $0.01)$, with no difference across groups for neutral object discrimination $\left(t_{(48)}=0.14, P=0.89\right)$. Within groups, a paired $t$-test confirmed enhanced negative relative to neutral discrimination in the stress group $\left(t_{(27)}=2.4, P=0.02\right)$, with no such difference in the control group $\left(t_{(21)}=-0.87, P=0.4\right)$. We also conducted the same analyses with sex as an additional factor and found no significant main effects or interactions with sex (all Ps >0.05), although we note that the study was not powered to detect sex differences. In the control group, there were 8 males and 14 females. In the stress group, there were 13 males and 15 females. Thus, our null findings are likely a result of small sample sizes and should not be taken as evidence of no sex differences. Together, these results suggest that stress selectively enhances negative object discrimination.

\section{General recognition enhancement for negative objects}

To examine whether, in addition to discrimination, stress affects general recognition memory of negative and neutral objects, we conducted a 2 (Emotion: Negative vs. Neutral) $\times 2$ (Group: Stress vs. Control) ANOVA, with Emotion as a repeated factor on the calculated d' scores (see Memory Analysis) for each valence. We found a significant main effect of emotion $\left(F_{(1,48)}=17.6, P<0.001\right.$, partial $\eta^{2}=0.27$, observed power $=0.98$; Fig. 3 ), where negative objects were better remembered than neutral objects. There was no main effect of group or interaction between emotion and group, although we note that the interaction was trending toward significance $\left(F_{(1,48)}=3.50, P=0.068\right)$. This is consistent with previous findings of emotional memory enhancements in general, and suggests that while stress selectively enhances negative discrimination in this task, it does not enhance general recognition. We also conducted the same analyses with sex as an additional factor and found no significant main effects or interactions with sex (all $P$ s $>0.05$ ). See Table 1 for raw proportion of responses (Old, Similar, New) for negative and neutral objects in the control and stress groups. We also tabulated the proportion of responses for the background stimuli (Table 2) and found no significant differences across groups or conditions.

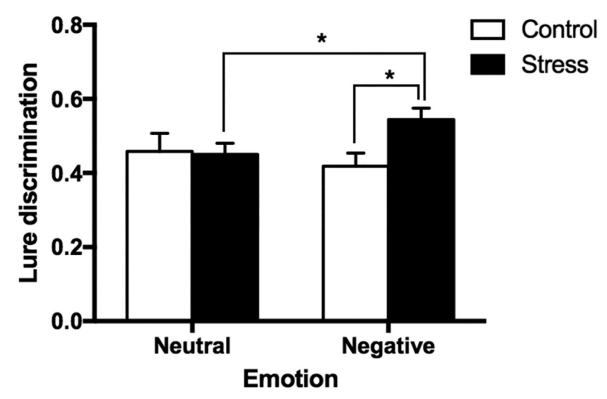

Figure 2. Lure discrimination in Control and Stress groups for Negative vs. Neutral objects. Lure discrimination [ $p$ ("Similar or New"|Lure)$\mathrm{p}$ ("Similar or New"|Target)] for negative and neutral objects in the stress group compared to controls. Error bars represent SEM.

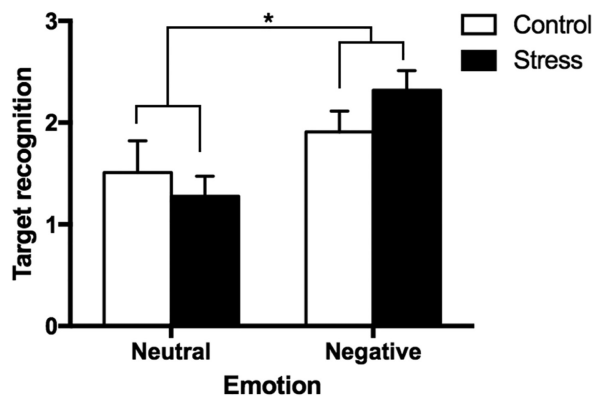

Figure 3. Target recognition in Control and Stress groups for Negative versus Neutral objects. Target recognition $[z($ Hits $)-z$ (False Alarms)] for negative and neutral objects in the stress group compared to controls. Error bars represent SEM.

\section{Relationship between cortisol and mnemonic discrimination}

In addition to exploring the impact of stress exposure on mnemonic discrimination, we were also interested in whether cortisol concentration would be related to changes in mnemonic discrimination performance. As noted above, the stress group showed a significantly greater cortisol increase following the TSST as measured by the greatest increase in salivary cortisol concentration post-stressor. In the following analyses, we included all participants, regardless of stress or control group status, in correlations between cortisol and performance to increase power. The maximum increase in salivary cortisol concentration was used as the independent variable with both negative and neutral LDI measures as the dependent variables. Linear analyses revealed no significant correlations between cortisol concentration and either negative $(r(50)=$ $0.16, P=0.26)$ or neutral $(r(50)=-0.04, P=0.77)$ LDI. Critically, however, when using a quadratic model in a curvilinear regression, we found a significant inverted $U$-shaped quadratic fit between cortisol concentration and negative $\operatorname{LDI}\left(F_{(2,47)}=3.7, P=0.03,95 \% \mathrm{CI}\right.$ $[-0.64,-0.064]$; Fig. 4A). The model did not fit the relationship between cortisol and neutral information $\left(F_{(2,47)}=0.2, P=0.8\right.$; Fig. $4 \mathrm{~b})$. When comparing the fits of the curves, the quadratic relationship between negative LDI and cortisol concentration was a better fit ( $\mathrm{AICc}=-174.3$ ) compared the quadratic relationship between neutral LDI and cortisol concentration (AICc $=-158)$. The probability the quadratic model is correct (compared to the linear model) is $86.29 \%$ for the negative LDI-cortisol relationship and only $26.54 \%$ for the neutral LDI-cortisol relationship. This finding falls directly in line with the Yerkes-Dodson inverted U-shaped dose-response model of stress (Yerkes and Dodson 1908). Importantly, it suggests that an increasing stress response, as measured by cortisol reactivity, selectively benefits mnemonic discrimination of emotionally negative information up to a moderate level, but once cortisol exceeds that level it begins to hinder performance.

\section{Discussion}

The primary goal of this study was to determine the effects of postencoding stress on the quality and discriminability of negative emotional versus neutral memory. Research has shown that stress has differential impacts on memory depending on when it is administered (e.g., de Quervain et al. 2009). Post-encoding manipulations have shown to be powerful in examining effects on consolidation. The importance of the post-encoding manipulation in understanding consolidation is to remove any impact the manipulation could have on encoding processes, making it possible 
Stress enhances negative discrimination

Table 1. Proportion of Old, Similar, and New responses to negative and neutral targets and lures in control and stress groups. Scores reported as means with SEM

\begin{tabular}{|c|c|c|c|c|c|c|}
\hline & \multicolumn{3}{|c|}{ Negative } & \multicolumn{3}{|c|}{ Neutral } \\
\hline & Old & Similar & New & Old & Similar & New \\
\hline \multicolumn{7}{|c|}{ Responses to target objects } \\
\hline $\begin{array}{l}\text { Control } \\
\text { Stress }\end{array}$ & $\begin{array}{l}0.67(0.03) \\
0.75(0.03)\end{array}$ & $\begin{array}{l}0.21(0.03) \\
0.16(0.01)\end{array}$ & $\begin{array}{l}0.12(0.03) \\
0.09(0.02)\end{array}$ & $\begin{array}{l}0.64(0.05) \\
0.64(0.03)\end{array}$ & $\begin{array}{l}0.15(0.03) \\
0.13(0.02)\end{array}$ & $\begin{array}{l}0.21(0.04) \\
0.23(0.03)\end{array}$ \\
\hline \multicolumn{7}{|c|}{ Responses to similar lure objects } \\
\hline $\begin{array}{l}\text { Control } \\
\text { Stress }\end{array}$ & $\begin{array}{l}0.25(0.04) \\
0.20(0.03)\end{array}$ & $\begin{array}{l}0.49(0.04) \\
0.53(0.03)\end{array}$ & $\begin{array}{l}0.25(0.04) \\
0.27(0.03)\end{array}$ & $\begin{array}{l}0.18(0.03) \\
0.19(0.02)\end{array}$ & $\begin{array}{l}0.41(0.04) \\
0.39(0.03)\end{array}$ & $\begin{array}{l}0.40(0.04) \\
0.42(0.04)\end{array}$ \\
\hline
\end{tabular}

to tease apart stress effects on encoding versus consolidation. Rodent studies have shown that drug injections or brain stimulation after learning can enhance memory consolidation (Gold and McGaugh 1975; Packard et al. 1994; McGaugh 2000). In humans, Cahill and colleagues showed that post-encoding stress (elicited via a cold-pressor task) enhanced memory for emotional arousing slides compared to controls but did not affect memory for neutral slides (Cahill et al. 2003).

In the present study, we exposed participants to the stressor after encoding. We aimed to address the question of whether stress enhances the long-term storage of precise emotional information or if it promotes a more general type of storage. We show that postencoding stress (via the TSST) resulted in selective enhancement of negative detailed information. For target recognition, we found a general emotion effect, where both stress and control groups showed better memory for negative versus neutral information. However, for mnemonic discrimination, we found an emotion effect that was specific to individuals who experienced postencoding stress; negative lure objects were more likely to be correctly distinguished as similar or new compared to neutral lure objects. Therefore, stress seems to selectively enhance the ability to discriminate negative information. Furthermore, similar to other research exploring the impact of endogenous cortisol release on memory performance (Andreano and Cahill 2006), analysis of salivary cortisol concentrations revealed an inverted $U$ effect, where those with moderate levels of cortisol showed enhanced mnemonic discrimination of negative stimuli, while those with low or high levels of salivary cortisol were worse at discriminating negative stimuli. This was not true for neutral stimuli, highlighting the specificity of the impact of cortisol on discrimination in that it only effects what is deemed emotionally salient, and thus, most adaptive to discriminate. Clearly, post-encoding stress led to highly accurate and detailed storage of emotional information. Moreover, stress selectively enhanced discrimination of negative stimuli.

The mechanism by which stress influences memory consolidation has been examined extensively over the years. During stress, epinephrine and glucocorticoids (such as cortisol) are released and influence the modulation of memory via a downstream release of norepinephrine in the basolateral amygdala which can influence hippocampal function (McGaugh 2004). Increased norepinephrine aids in remembering an emotional event and when further interacting with cortisol can lead to optimal strengthening of memories (Joëls et al. 2011). While many human studies have shown that stress increases memory for emotionally arousing information (e.g., Payne and Nadal 2004; Payne et al. 2007), few studies have explored how stress affects the specificity of memory.

Prior work has shown that presenting participants with a set of emotionally arousing images immediately before they encode everyday neutral objects is associated with noradrenergic activation as measured by salivary $\alpha$-amylase, which predicts mnemonic discrimination performance (Segal et al. 2012). However, this study only evaluated emotional arousal as a prestudy state effect, in which an increased state of arousal could have enhanced attention or increased vigilance rather than discrimination ability. Our findings suggest that activation of the HPA axis leading to the release of cortisol during the early consolidation period is associated with an impact on the ability to discriminate emotional memories.

Another study used an emotional mnemonic discrimination task to test participants' memory in discriminating similar negative, neutral, and positive scenes. Unlike the aforementioned design, this task did not use an arousal-inducing prestudy state, but rather examined transient fluctuations in arousal and their impact on memory (Leal et al. 2014a). The authors demonstrated an impairment in mnemonic discrimination for emotional versus neutral items, but a preservation of general recognition for emotional versus neutral items, which is consistent with previously reported gist versus detail trade-offs in emotional memory (Loftus et al. 1987; Buchanan and Adolphs 2002; Kensinger 2009; Mather and Sutherland 2011). High-resolution functional MRI revealed greater hippocampal dentate gyrus (DG) and CA3 activity specifically during emotional versus neutral discrimination (Leal et al. 2014a) and greater amygdala activity on emotional versus neutral trials regardless of accurate memory performance, suggesting two dissociable roles for the amygdala (emotional modulation) and the hippocampus (mnemonic discrimination). Based on these results as well as the large literature on the role of the amygdala in enhancing

Table 2. Proportion of Old, Similar, and New responses to negative and neutral target backgrounds and lure backgrounds in control and stress groups. Scores reported as Means with SEM

\begin{tabular}{|c|c|c|c|c|c|c|}
\hline & \multicolumn{3}{|c|}{ Negative } & \multicolumn{3}{|c|}{ Neutral } \\
\hline & Old & Similar & New & Old & Similar & New \\
\hline \multicolumn{7}{|c|}{ Responses to target backgrounds } \\
\hline $\begin{array}{l}\text { Control } \\
\text { Stress }\end{array}$ & $\begin{array}{l}0.45(0.04) \\
0.41(0.03)\end{array}$ & $\begin{array}{l}0.17(0.03) \\
0.17(0.03)\end{array}$ & $\begin{array}{l}0.38(0.03) \\
0.42(0.03)\end{array}$ & $\begin{array}{l}0.54(0.04) \\
0.55(0.03)\end{array}$ & $\begin{array}{l}0.16(0.02) \\
0.17(0.02)\end{array}$ & $\begin{array}{l}0.30(0.04) \\
0.28(0.03)\end{array}$ \\
\hline \multicolumn{7}{|c|}{ Responses to lure backgrounds } \\
\hline $\begin{array}{l}\text { Control } \\
\text { Stress }\end{array}$ & $\begin{array}{l}0.17(0.03) \\
0.16(0.02)\end{array}$ & $\begin{array}{l}0.27(0.02) \\
0.28(0.03)\end{array}$ & $\begin{array}{l}0.56(0.04) \\
0.56(0.04)\end{array}$ & $\begin{array}{l}0.17(0.03) \\
0.14(0.02)\end{array}$ & $\begin{array}{l}0.30(0.04) \\
0.28(0.03)\end{array}$ & $\begin{array}{l}0.53(0.04) \\
0.58(0.04)\end{array}$ \\
\hline
\end{tabular}


A

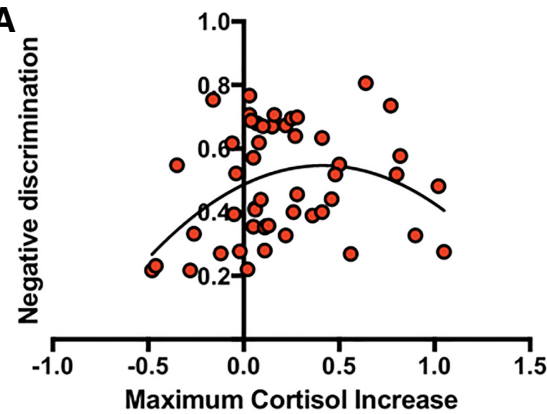

B

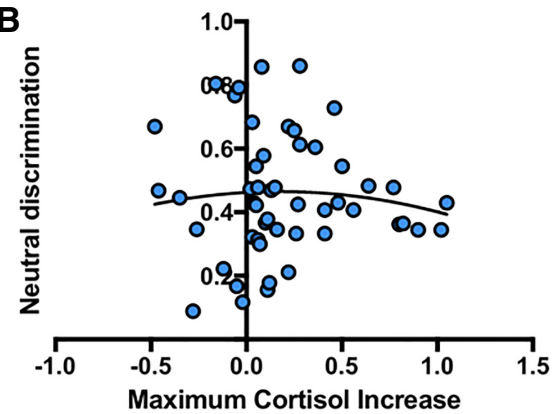

Figure 4. Relationship between negative and neutral lure discrimination and cortisol. ( $A$ ) Correlation between maximum cortisol increase (subtracting baseline cortisol concentrations (t0) from the maximum cortisol increase following the stressor) and negative lure discrimination. (B) Correlation between maximum cortisol increase and neutral lure discrimination.

affective memory consolidation (see McGaugh 2004), we suggest that the cascade of cortisol and other neuromodulators (e.g., norepinephrine) following exposure to stress leads to enhanced amygdala activation, which in turn may influence hippocampal DG/CA3 function when processing emotional stimuli. Thus, cortisol release after a stressful event may lead to stronger amygdala-DG/CA3 connectivity that enhances the ability to discriminate emotional information. However, our results also suggest that the relationship between stress and discrimination is nonlinear, exhibiting an inverted U-shaped curve.

Altered cortisol levels occur in many clinical disorders as well. For example, elevated cortisol levels are common in depression (Yehuda et al. 1996; Young et al. 2000; Herbert 2013), while lower cortisol levels have been found in post-traumatic stress disorder (PTSD) (Yehuda et al. 1996). Thus, another important question is whether acute versus chronic stress yields similar results. Previously, we reported enhanced discrimination of negative stimuli in individuals with depressive symptoms (Leal et al. 2014a), similar to the enhanced negative mnemonic discrimination we report here in individuals who are acutely stressed. As such, it raises the interesting possibility of overlapping mechanisms in acute and chronic stress conditions, although further research will be necessary to assess this given that elevated stress and cortisol secretion are not the only changes observed in depression. Nevertheless, it is interesting that individuals with depressive symptoms also show deficits in neutral discrimination compared to healthy controls (Leal et al. 2014a), while our healthy stressed participants demonstrated no difference in neutral discrimination relative to controls. While speculative at this point, this might suggest that acute and chronic stressors elicit similar enhanced memory processing of negative information, whereas chronic stress may have an additional impact on the discrimination of neutral information. Further, the enhancement effect in individuals with depressive symptoms appears to be specific to mnemonic discrimination, as previous studies found no influence on target recognition abilities (Leal et al. 2014a). Here, acutely stressed individuals demonstrate enhanced negative versus neutral target recognition in addition to enhanced mnemonic discrimination. However, controls also had greater target recognition for negative versus neutral objects, suggesting that individuals with chronically elevated HPA axis activation may have impaired target recognition while acute stress does not have this impact. Measuring memory for general target memory in addition to mnemonic discrimination leads to different interpretations of how stress affects memory, which highlights the importance of taking these differences into account.

Previously, high-resolution imaging performed in individuals with depressive symptoms revealed a shift in amygdalahippocampal dynamics, where depressed participants showed regist versus detail information are key to understanding the mechanisms underlying the effects of stress on hippocampal function and will be an important future direction.

One of the most exciting aspects of these results is that it opens the door to a host of new interesting questions for future research. To begin, further studies using neuroimaging will be necessary to link amygdala and hippocampal connectivity with levels of cortisol activity during negative discrimination to understand how these systems work together to result in greater discrimination of negative information. Efforts should also be made to elicit stress at different stages of memory formation to determine how stress administered at encoding and retrieval impact the discrimination of different kinds of memory.

A number of future studies should also be designed to assess some of the major limitations of this study. One important limitation of this study is that stress and arousal are associated with the release of a host of neuromodulators and both psychological and physiological responses. As such, the cortisol measured in this design is only a marker of stress effects. Further research is necessary to determine the specific contributions of cortisol, the influence of other neuromodulators such as norepinephrine released during stress and arousal, more widespread effects stress may have on the system, as well as the differing effects between acute elevations in cortisol and the chronically elevated cortisol seen in depression and other affective disorders. A second limitation is that, while trending, we did not find a significant effect of stress on target recognition memory for negative stimuli that has previously been reported in the literature (Payne and Nadel 2004; Payne et al. 2007). This may also be an issue of power, where with more subjects the effect may have reached significance. However, if we can pick up on stress effects for lure discrimination but not target recognition, it is also a possibility that lure discrimination measures may be more sensitive to the effects of stress. Another possibility is that the inclusion of similar stimuli during testing may also influence how target stimuli are remembered, so we may not expect to see the exact same results as previous studies that did not include this stimulus type in their paradigms. Future studies should address these possibilities in determining the differential effects of stress on lure discrimination and target recognition.

Another important direction for future research will be the use of imaging to determine the brain mechanisms behind the reported stress effects on mnemonic discrimination. While, as discussed above, a number of studies have found amplified activity in the basolateral amygdala and enhanced connectivity among the amygdala and other memory-relevant regions of the brain in response to stress and cortisol (McGaugh 2004; Roozendaal et al. 2009), other studies have found reduced amygdala activation following a psychosocial stressor (e.g., Pruessner et al. 2008). Additionally, a number of clinical studies have found that 
administration of exogenous cortisol leads to a reduction in typical symptomatology and fear behavior in individuals with anxiety (Putman et al. 2007), phobias (Soravia et al. 2006), and PTSD (Aerni et al. 2004), also indicating a cortisol may have alternate effects on subsequent amygdala activation and memory consolidation. Given that our explanation of the reported effects is highly reliant on the theory that cortisol enhances amygdala activation and connectivity, our conclusions will remain speculative until validated by further research. Another limitation of this study is the lack of power to detect an influence of sex on performance under our task conditions. Future investigation is necessary to understand possible sex differences in mnemonic discrimination under stressful conditions. In addition, mnemonic discrimination tasks that include a three-response system such as the one used in the current study (i.e., Old, Similar, New) can examine lure discrimination in multiple ways, such as only looking at a "Similar" response to a lure versus combining "Similar" and "New" responses, whereas a two-response system (i.e., Old, New) is less open to interpretation, since "New" is the only correct response for discriminating lures. A limitation of the three-response system is that a "New" response to a lure could mean discrimination or forgetting. Although we did correct for response bias, it does not entirely eliminate the potential problem and additional means of examining mnemonic discrimination should be explored in future studies. Finally, in the present study we only focused on negative (and not positive) emotional stimuli. We chose to focus on negative stimuli as these types of stimuli typically elicit universally greater arousal compared to neutral stimuli, while positive stimuli are more influenced by subjective interpretation and prior experience (e.g., a photo of a puppy for a dog lover compared to someone that is allergic to dogs). Future studies should include positive stimuli to examine how stress may impact memory of positive items as well. If positive stimuli were successful at generating the same level of arousal, then we would expect to see similar results for both positively and negatively valenced information.

To our knowledge, this study was the first to explore the impact of acute stress on the mnemonic discrimination of emotional and neutral stimuli. We find that the stress effect is specific to mnemonic discrimination, only for emotional stimuli. While several previous studies using general recall or recognition scores show typical emotion-related enhancements in memory following stress (replicated here), the discrimination measure allows us to better tease apart stress-related effects on emotional memory. Our findings suggest that rather than leading to a blanket enhancement in emotional memory, moderate stress may be playing a more important role in distinguishing previously experienced emotional experiences from one another. It will be vital for future researchers to continue to explore the relationship between stress and discrimination abilities, as this information is important for understanding both everyday experiences and the unique memory patterns found in a variety of clinical populations.

\section{Materials and Methods}

\section{Participants}

Fifty participants (29 female) from the University of Notre Dame (mean age $18.9 \pm 0.9$ ), who were part of a larger ongoing study on sleep-stress interactions, were tested in two experimental sessions, saw the day before.
$1 \mathrm{~d}$ apart. They participated for payment or course credit. Participants had normal or corrected-to-normal vision and English was their primary language. Prior to enrollment in the study, they were screened to ensure that they were free of sleep disorders, history of psychiatric illness (including anxiety or mood disorders), and the use of medication affecting the endocrine or central nervous systems. Informed consent was obtained from all participants, with all procedures approved by the University of Notre Dame Institutional Review Board.

\section{Materials}

Each scene used during the encoding session depicted a negative arousing, or neutral object on a plausible neutral background (Kensinger et al. 2007; Payne et al. 2008). For each of 64 scenes (e.g., a car on a street), eight different versions were created by placing two similar neutral objects (e.g., two images of a car) and two similar negative objects (e.g., two images of a car crash) on two neutral backgrounds (e.g., two images of a street; see Fig. 5). An additional 32 completely new scenes served as foils on the subsequent recognition memory test. The objects and backgrounds were previously normed on valence and arousal using 7-point Likert scales (Kensinger et al. 2006). All negative objects received valence ratings $<3(1=$ most negative $)$ and arousal ratings of 5-7 ( 7 = most arousing), while neutral items (both objects and backgrounds) were rated neutral in valence (between 3 and 5) and nonarousing (arousal values $<4$ ). Target objects and their similar lures were previously matched based on size, overall similarity, dimensions, and familiarity, and were placed in the same approximate location on the scenes. Importantly, final pairs were selected such that emotional pairs and neutral pairs also did not differ in any of these categories (Kensinger et al. 2007).

\section{Experimental design}

Participants were randomly assigned to either a stress $(N=28,15 \mathrm{fe}-$ male, mean age $18.8 \pm 0.9)$ or control group $(N=22,14$ female, mean age $18.9 \pm 0.9)$. The study commenced in the late afternoon (between 4 and 5 p.m.) to control for circadian influences on cortisol response. Upon arrival, participants completed paperwork for $20 \mathrm{~min}$ while acclimating to the laboratory setting. After this acclimation period, they provided their first saliva sample. Participants were instructed to use the passive drool technique using a straw to expectorate (i.e., no cotton, gum, or other saliva flow stimulants were used) and were asked to fill the test tube to the $5 \mathrm{~mL}$

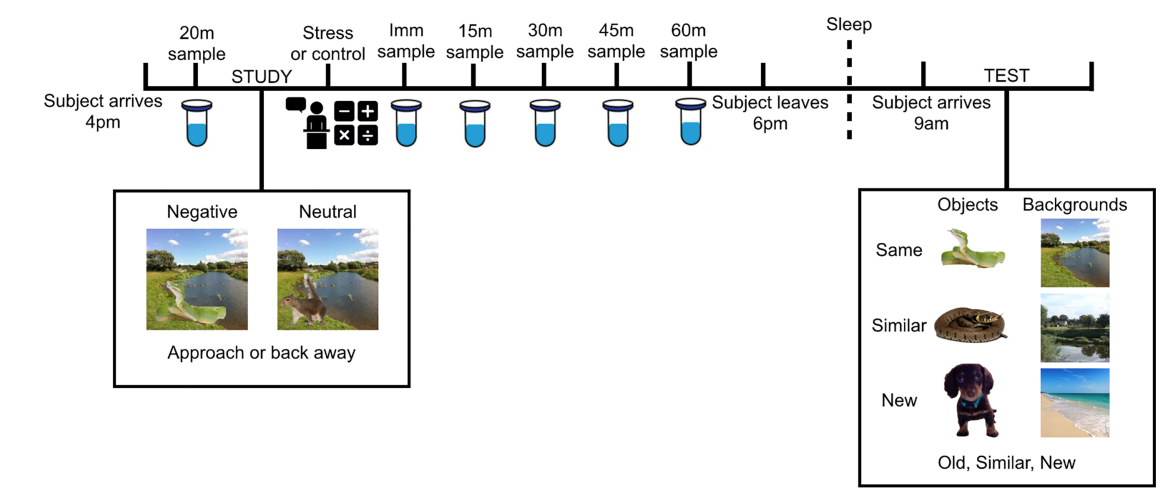

Figure 5. Experimental procedure. The subject arrives at 4 p.m. and takes a saliva sample after 20 min $(20 \mathrm{~m})$. They then complete the incidental study session in which they are shown scenes with objects embedded in them (either negative or neutral) and are asked if they would approach or avoid the scene. Immediately following encoding, subjects either undergo the TSST or the control condition, which involves giving a speech and doing a math task (see Materials and Methods for details). Saliva samples are collected immediately after the stressor/control manipulation followed by saliva samples $15,30,45$, and 60 min post-stress. The subject leaves the lab and returns at 9 a.m. the following day and completes the recognition test, where they are presented with objects and backgrounds separately and are asked to determine if they are the same, similar, or new objects/backgrounds from what they 
line for each sample. They were allowed to drink sips of water during the session, but only immediately after a sample was completed.

After the acclimation period and initial sample, each participant completed the encoding task, which consisted of viewing 64 scenes (32 with a negative central object and 32 with a neutral central object, all placed on neutral backgrounds) for $5 \mathrm{sec}$ each. For each scene, participants used a 7-point scale to indicate whether they would approach or back away from the scene if they were to encounter it in real life. This task was used to ensure that participants were paying attention to the scenes and to promote deep encoding (Waring et al. 2010). The encoded version of each scene (of the eight possible versions) was counterbalanced across participants.

After encoding, participants were escorted to a separate room and exposed to a validated psychosocial stressor, the TSST (Kirschbaum et al. 1993), or a matched control treatment (see below), both of which lasted $\sim 20 \mathrm{~min}$. The TSST is a well-established method of stress induction, reliably inducing HPA activation in laboratory settings (Kirschbaum et al. 1993). It combines stressor uncontrollability with social evaluative stress, which together produce a large cortisol response in humans (Dickerson and Kemeny 2004). Participants completing the TSST were told that they would be judged on verbal and nonverbal performance while delivering a speech. They were then given a 10-min preparation period to write a speech about why they would be the best candidate for a job position. The presentation could be on any job position, but they were required to use only truthful information about themselves and were not allowed to fabricate details. The participants took notes during the preparation period, but the notes were abruptly taken away from participants just before they began their speech. Participants then delivered their speech for 5 min standing in front of two seated judges wearing lab coats. The participants were also given the impression that their performance was being videoand audio-recorded for later analysis. If the participants finished before $5 \mathrm{~min}$, they were instructed to continue until the full 5 min elapsed. Immediately following the speech, participants were instructed to perform an out loud arithmetic task (subtract 13 's from 1022 continuously, and as quickly and accurately as possible for another $5 \mathrm{~min}$ ). If they made a mistake, they were told, "No, that is not correct. Start over from 1022." Once the math task was over, they were dismissed to return to the experimenter.

Participants in the control condition also prepared the speech, but prior to doing so were informed that they were in the control condition and would not be presenting it in front of anyone. Instead, after preparation, they sat alone in an empty room and read their speech aloud from their notes while seated with no audio or video equipment present. They were then asked to complete an easier math task (subtracting 15's from 1000), again with no one directly observing them. Participants were surreptitiously observed through a window to ensure that they followed these instructions and remained awake.

A total of six saliva samples were collected throughout the course of the initial encoding session: (t0) a baseline sample collected 20 min after participant arrival, (t1) immediately after completion of the stress/control task, (t2) $15 \mathrm{~min}$ after stress/control task, (t3) $30 \mathrm{~min}$ after stress/control task, and (t4) 45 min after stress/control task, and (t5) $60 \mathrm{~min}$ after stress/control task. Measures of state anxiety (STAI, Spielberger et al. 1983) and affect (PANAS, Watson et al. 1988) were also collected at baseline prior to the encoding task, immediately after the stress/control manipulation, and again the next day prior to recognition testing. Following the final saliva sample, participants were instructed to return the next day for a follow-up session and were dismissed.

The following day, participants returned to the lab at 9 a.m. to perform an unexpected, self-paced recognition task. During this task, objects and backgrounds from the encoded scenes were presented individually and one at a time. The recognition task was composed of scene components that were identical to the objects and backgrounds that had been encoded (e.g., the same car crash), alternate versions of the object or background (i.e., shared the same verbal label but differed in specific visual details; a similar car accident), and other objects and backgrounds that had not been previ- ously viewed (completely new). Participants either saw the same or the similar version of a particular item at test, never both. For each item, participants were asked to determine whether it was identical to a previously viewed scene component ("same"), similar but not an exact match ("similar"), or not seen during encoding ("new").

The recognition task included 32 same objects (16 negative, 16 neutral), 32 similar objects (16 negative, 16 neutral), 32 new objects (16 negative, 16 neutral), 32 same backgrounds (16 previously presented with a negative object, 16 previously presented with a neutral object), 32 similar backgrounds (16 previously presented with a negative object, 16 previously presented with a neutral object), and 32 new backgrounds.

\section{Cortisol reactivity assessment}

After each session, vials were capped and frozen until later processing. Radioimmunoassays were done in the on-campus wet lab at Notre Dame and began with three freeze-thaw cycles and centrifugation to reduce viscosity and remove salivary debris. The protocol described by Wirth and Schultheiss (2006) was used to determine cortisol levels through solid-phase ${ }^{125}$ I radioimmunoassays. In total, seven assays were necessary to complete all the samples. The study required ongoing data collection and processing. Following the first four assays the initial assay kit provider discontinued their product (Coat-A-Count, Siemens Healthcare Diagnostics, Duluth, GA). This required the use of a new provider for the final three assays (MP Biomedicals, Santa Ana, CA). Samples with an initial CV greater than 60 in both kits were reassayed using their original kits. Samples were then $Z$-scored within each kit to create standardized cortisol concentrations. Fifty-one samples from the original kits were randomly selected to be assayed again using the MP Biomedicals kit. Comparison of the standardized $Z$-scores revealed a high correlation between kits $(r(51)=0.73, P<0.0001)$, verifying the validity of this approach.

After removing samples under the lower limit of detection (LLD), the mean intra-assay coefficient of variation (CV) was 19.9. Inter-assay CVs for Morning and Evening combined pools of saliva averaged 12.2 and 16.0, respectively. The average LLD $\left(\mathrm{B}_{0}-3 \times \mathrm{SD}\right.$ method) was $0.21 \mathrm{ng} / \mathrm{mL}$. External controls tested at expected values.

\section{Memory analysis}

We focused our analyses on how well participants remembered negative and neutral objects. To do so, we examined performance using a response bias-corrected LDI measure previously used in studies of mnemonic discrimination (Leal et al. 2014a, 2014b; Marks et al. 2017). In order to measure how well participants discriminated similar objects (lures) from old and new images, we operationalized LDI for this study as p("Similar or New"|Lure)$\mathrm{p}$ ("Similar or New"|Target). This corrected for the general tendency to reject (i.e., call an item "Similar" or "New") and has been used in prior work (Marks et al. 2017). This score captures the ability to discriminate similar items by giving a "similar" or "new" response, in which participants had to remember some specific component of the object in order to reject it. These responses can be viewed as different thresholds for detecting differences among stimuli but are generally classified as items that were not falsely recognized (i.e., 1 minus p("Old"|Lure). While "similar" is the most correct response to a lure item and has been used alone as a measure of lure discrimination (Yassa et al. 2011; Stark et al. 2013), a "new" response may also reflect the accurate rejection of a lure item as old (Marks et al. 2017), thus we combined these responses to measure correct rejections of lure stimuli.

Consistent with prior studies, a general memory recognition score was also computed by summing the number of "same" responses to same items. Target recognition was measured by a discriminability index $\left(\mathrm{d}^{\prime}\right)$, which was calculated as $\mathrm{z}$ ("Old"|Target) z("Old"|Foil), which is thought to assess gist knowledge or general familiarity (Norman 2010; Yonelinas et al. 2010). Both of these measures allow us to investigate memory, but for unique trial types (i.e., target recognition versus mnemonic discrimination). 


\section{Statistical analysis}

All statistical analyses were conducted in SPSS v. 24 (IBM Corp., Armonk, NY). Planned comparisons were conducted using repeated-measures ANOVAs. Post-hoc contrasts were conducted where appropriate. All tests used the General Linear Model (ANOVA and correlations). Normality assumptions were investigated using Kolmogorov-Smirnov tests and all distributions investigated did not significantly deviate from the normal distribution. Repeated measures tests were corrected for error nonsphericity using Greenhouse-Geisser correction where appropriate. The Akaike information criterion (AIC) was used to compare which curve fitting approach fitted the data best. Statistical values were considered significant at a final corrected $\alpha$ level of 0.05 , which appropriately controls for Type I error.

\section{Acknowledgments}

This work was supported by a grant from the National Science Foundation BCS-0963581 to J.D.P. Support for M.A.Y. was provided by National Institutes of Health grants by R01 AG053555, R21 AG049220, P50 AG16573, and R01 MH102392. S.L.L. was supported by an NIA F32 AG054116.

Competing interest statement: The authors declare no competing financial interests.

\section{References}

Abercrombie HC. 2009. Hypothalamic-pituitary-adrenal axis. In The international encyclopedia of depression (ed. Ingram RE), pp. 332-336. Springer Publishing Company, New York, NY.

Abercrombie H, Speck N, Monticelli R. 2006. Endogenous cortisol elevations are related to memory facilitation only in individuals who are emotionally aroused. Psychoneuroendocrinology 31: 187-196.

Aerni A, Traber R, Hock C, Roozendaal B, Schelling G, Papassotiropoulos A, Nitsch RM, Schnyder U, de Quervain DJ. 2004. Low-dose cortisol for symptoms of posttraumatic stress disorder. Am J Psychiatry 161: 1488-1490.

Andreano JM, Cahill L. 2006. Glucocorticoid release and memory consolidation in men and women. Psychol Sci 17: 466-470.

Buchanan TW, Adolphs R. 2002. The role of the human amygdala in emotional modulation of long-term declarative memory. In Emotional cognition from brain to behaviour (ed. Moore SC, Oaksford M), pp. 9-34. John Benjamins Publishing Company, Amsterdam.

Buchanan TW, Lovallo WR. 2001. Enhanced memory for emotional material following stress-level cortisol treatment in humans. Psychoneuroendocrinology 26: 307-317.

Cahill L, Gorski L, Le K. 2003. Enhanced human memory consolidation with post-learning stress: interaction with the degree of arousal at encoding. Learn Mem 10: 270-274.

Cunningham TJ, Crowell CR, Alger SE, Kensinger EA, Villano MA, Mattingly SM, Payne JD. 2014. Psychophysiological arousal at encoding leads to reduced reactivity but enhanced emotional memory following sleep. Neurobiol Learn Mem 114: 155-164.

de Quervain DJ, Aerni A, Schelling G, Roozendaal B. 2009. Glucocorticoids and the regulation of memory in health and disease. Front Neuroendocrinol 30: 358-370.

Déry N, Pilgrim M, Gibala M, Gillen J, Wojtowicz JM, Macqueen G, Becker S. 2013. Adult hippocampal neurogenesis reduces memory interference in humans: opposing effects of aerobic exercise and depression. Front Neurosci 7: 66 .

Dickerson SS, Kemeny ME. 2004. Acute stressors and cortisol responses: a theoretical integration and synthesis of laboratory research. Psychol Bull 130: 355-391.

Fujii T, Saito DN, Yanaka HT, Kosaka H, Okazawa H. 2014. Depressive mood modulates the anterior lateral CA1 and DG/CA3 during a pattern separation task in cognitively intact individuals: a functional MRI study. Hippocampus 24: 214-224.

Gold PE, McGaugh JL. 1975. A single-trace, two process view of memory storage processes. In Short-term memory (ed. Deutsch D, Deutsch JA), pp. 355-378. Academic Press, New York, NY.

Herbert J. 2013. Cortisol and depression: three questions for psychiatry. Psychol Med 43: 449-469.

Joëls M, Fernandez G, Roozendaal B. 2011. Stress and emotional memory: a matter of timing. Trends Cogn Sci 15: 280-288.

Kajantie E, Phillips D. 2006. The effects of sex and hormonal status on the physiological response to acute psychosocial stress. Psychoneuroendocrinology 31: 151-178.
Kensinger EA. 2009. Remembering the details: effects of emotion. Emot Rev 1: 99-113.

Kensinger EA, Garoff-Eaton RJ, Schacter DL. 2006. Memory for specific visual details can be enhanced by negative arousing content. J Mem Lang 54: $99-112$.

Kensinger EA, Garoff-Eaton RJ, Schacter DL. 2007. Effects of emotion on memory specificity: memory trade-offs elicited by negative visually arousing stimuli. J Mem Lang 56: 575-591.

Kirschbaum C, Pirke KM, Hellhammer DH. 1993. The "Trier Social Stress Test" - a tool for investigating psychobiological stress responses in a laboratory setting. Neuropsychobiology 28: 76-81.

Kudielka BM, Kirschbaum C. 2005. Sex differences in HPA axis responses to stress: a review. Biol Psychol 69: 113-132.

Kudielka BM, Hellhammer DH, Kirschbaum CHJ. 2007. Ten years of research with the Trier Social Stress Test-revisited. Guilford Press, New York, NY.

Leal SL, Yassa MA. 2018. Integrating new findings and examining clinical applications of pattern separation. Nat Neurosci 21: $163-173$.

Leal SL, Tighe SK, Yassa MA. 2014a. Asymmetric effects of emotion on mnemonic interference. Neurobiol Learn Mem 111: 41-48.

Leal SL, Tighe SK, Jones CK, Yassa MA. 2014b. Pattern separation of emotional information in hippocampal dentate and CA3. Hippocampus 24: $1146-1155$.

Loftus EF, Loftus GR, Messo J. 1987. Some facts about "weapon focus." Law Hum Behav 11: 55-62.

Marks SM, Lockhart SN, Baker SL, Jagust WJ. 2017. Tau and $\beta$-amyloid are associated with medial temporal lobe structure, function and memory encoding in normal aging. J Neurosci 37: 3192-3201.

Marr D. 1971. Simple memory: a theory for archicortex. Philos Trans R Soc Lond B Biol Sci 262: 23-81.

Mather M, Sutherland MR. 2011. Arousal-biased competition in perception and memory. Perspect Psychol Sci 6: 114-133.

McClelland JL, McNaughton BL, O'Reilly RC. 1995. Why there are complementary learning systems in the hippocampus and neocortex: insights from the successes and failures of connectionist models of learning and memory. Psychol Rev 102: 419-457.

McGaugh JL. 2000. Memory-a century of consolidation. Science 287: 248-251.

McGaugh JL. 2004. The amygdala modulates the consolidation of memories of emotionally arousing experiences. Annu Rev Neurosci 27: 1-28.

McIntyre CK, McGaugh JL, Williams CL. 2012. Interacting brain systems modulate memory consolidation. Neurosci Biobehav Rev 36: 1750-1762.

Nielsen SE, Ahmed I, Cahill L. 2013. Sex and menstrual cycle phase at encoding influence emotional memory for gist and detail. Neurobiol Learn Mem 106: 56-65.

Norman KA. 2010. How hippocampus and cortex contribute to recognition memory: revisiting the complementary learning systems model. Hippocampus 20: 1217-1227.

Otte C, Hart S, Neylan TC, Marmar CR, Yaffe K, Mohr DC. 2005. A meta-analysis of cortisol response to challenge in human aging: importance of gender. Psychoneuroendocrinology 30: 80-91.

Packard MG, Cahill L, McGaugh JL. 1994. Amygdala modulation of hippocampal-dependent and caudate nucleus-dependent memory processes. Proc Natl Acad Sci 91: 8477-8481.

Pariante CM, Miller AH. 2001. Glucocorticoid receptors in major depression: relevance to pathophysiology and treatment. Biol Psychiatry 49: 391-404.

Payne JD, Nadel L. 2004. Sleep, dreams, and memory consolidation: the role of the stress hormone cortisol. Learn Mem 11: 671-678.

Payne JD, Jackson ED, Ryan L, Hoscheidt S, Jacobs JW, Nadel L. 2006. The impact of stress on neutral and emotional aspects of episodic memory. Memory 14: 1-16.

Payne JD, Jackson ED, Hoscheidt S, Ryan L, Jacobs WJ, Nadel L. 2007. Stress administered prior to encoding impairs neutral but enhances emotional long-term episodic memories. Learn Mem 14: 861-868.

Payne JD, Stickgold R, Swanberg K, Kensinger EA. 2008. Sleep preferentially enhances memory for emotional components of scenes. Psychol Sci 19: 781-788.

Pruessner JC, Dedovic K, Khalili-Mahani N, Engert V, Pruessner M, Buss C, Renwick R, Dagher A, Meaney MJ, Lupien S. 2008. Deactivation of the limbic system during acute psychosocial stress: evidence from positron emission tomography and functional magnetic resonance imaging studies. Biol Psychiatry 63: 234-240.

Putman P, Hermans EJ, Koppeschaar H, van Schijndel A, van Honk J. 2007. A single administration of cortisol acutely reduces preconscious attention for fear in anxious young men. Psychoneuroendocrinology 32: 793-802.

Roberson-Nay R, McClure EB, Monk CS, Nelson EE, Guyer AE, Fromm SJ, Charney DS, Leibenluft E, Blair J, Ernst M, et al. 2006. Increased amygdala activity during successful memory encoding in adolescent major depressive disorder: an fMRI study. Biol Psychiatry 60: 966-973.

Roozendaal B, McEwen BS, Chattarji S. 2009. Stress, memory and the amygdala. Nat Rev Neurosci 10: 423-433. 
Segal SK, Stark SM, Kattan D, Stark CE, Yassa MA. 2012. Norepinephrinemediated emotional arousal facilitates subsequent pattern separation. Neurobiol Learn Mem 97: 465-469.

Shapiro M, Olton D. 1994. Hippocampal function and interference. In What are the memory systems of 1994? (ed. Schacter DL, Tulving E), pp. 87-117. MIT Press, Cambridge, MA.

Shelton DJ, Kirwan CB. 2013. A possible negative influence of depression on the ability to overcome memory interference. Behav Brain Res 256: 20-26.

Shields GS, Sazma MA, McCullough AM, Yonelinas AP. 2017. The effects of acute stress on episodic memory: a meta-analysis and integrative review. Psychol Bull 143: 636-675.

Siegle GJ, Steinhauer SR, Thase ME, Stenger VA, Carter CS. 2002. Can't shake that feeling: event-related fMRI assessment of sustained amygdala activity in response to emotional information in depressed individuals. Biol Psychiatry 51: 693-707.

Siegle GJ, Thompson W, Carter CS, Steinhauer SR, Thase ME. 2007. Increased amygdala and decreased dorsolateral prefrontal BOLD responses in unipolar depression: related and independent features. Biol Psychiatry 61: 198-209.

Smeets T, Otgaar H, Candel I, Wolf OT. 2008a. True or false? Memory is differentially affected by stress-induced cortisol elevations and sympathetic activity at consolidation and retrieval. Psychoneuroendocrinology 33: 1378-1386.

Smeets T, Sijstermans K, Gijsen C, Peters M, Jelicic M, Merckelbach H. 2008 b. Acute consolidation stress enhances reality monitoring in healthy young adults. Stress 11: 235-245.

Soravia LM, Heinrichs M, Aerni A, Maroni C, Schelling G, Ehlert U, Roozendaal B, de Quervain DJ. 2006. Glucocorticoids reduce phobic fear in humans. Proc Natl Acad Sci 103: 5585-5590.

Spielberger CD, Gorsuch RL, Lushene PR, Vagg PR, Jacobs AG. 1983. Manual for the State-Trait Anxiety Inventory (Form Y). Consulting Psychologists Press, Palo Alto, CA.

Stark SM, Yassa MA, Lacy JW, Stark CE. 2013. A task to assess behavioral pattern separation (BPS) in humans: data from healthy aging and mild cognitive impairment. Neuropsychologia 51: 2442-2449.

Treves A, Rolls ET. 1992. Computational constraints suggest the need for two distinct input systems to the hippocampal CA3 network. Hippocampus 2: 189-199.

Vaisvaser S, Lin T, Admon R, Podlipsky I, Greenman Y, Stern N, Fruchter E, Wald I, Pine DS, Tarrasch R, et al. 2013. Neural traces of stress: cortisol related sustained enhancement of amygdala-hippocampal functional connectivity. Front Hum Neurosci 7: 313.

van Stegeren AH, Wolf OT, Everaerd W, Scheltens P, Barkhof F, Rombouts SA. 2007. Endogenous cortisol level interacts with noradrenergic activation in the human amygdala. Neurobiol Learn Mem 87: $57-66$.

Vyas A, Mitra R, Shankaranarayana Rao BS, Chattarji S. 2002. Chronic stress induces contrasting patterns of dendritic remodeling in hippocampal and amygdaloid neurons. J Neurosci 22: 6810-6818.

Waring JD, Payne JD, Schacter DL, Kensinger EA. 2010. Impact of individual differences upon emotion-induced memory trade-offs. Cogn Emot 24: 150-167.

Watson D, Clark LA, Tellegen A. 1988. Development and validation of brief measures of positive and negative affect: the PANAS scales. J Pers Soc Psychol 54: 1063-1070.

Wirth MM, Schultheiss OC. 2006. Effects of affiliation arousal (hope of closeness) and affiliation stress (fear of rejection) on progesterone and cortisol. Horm Behav 50: 786-795.

Wolf OT. 2009. Stress and memory in humans: twelve years of progress? Brain Res 1293: 142-154.

Yassa MA, Stark CE. 2011. Pattern separation in the hippocampus. Trends Neurosci 34: 515-525.

Yassa MA, Lacy JW, Stark SM, Albert MS, Gallagher M, Stark CE. 2011. Pattern separation deficits associated with increased hippocampal CA3 and dentate gyrus activity in nondemented older adults. Hippocampus 21: $968-979$.

Yehuda R, Teicher MH, Trestman RL, Levengood RA, Siever LJ. 1996. Cortisol regulation in posttraumatic stress disorder and major depression: a chronobiological analysis. Biol Psychiatry 40: 79-88.

Yerkes RM, Dodson JD. 1908. The relation of strength of stimulus to rapidity of habit-formation. J Comp Neurol Psychol 18: 459-482.

Yonelinas AP, Aly M, Wang WC, Koen JD. 2010. Recollection and familiarity: examining controversial assumptions and new directions. Hippocampus 20: 1178-1194.

Young E, Lopez JF, Murphy-Weinberg V, Watson SJ, Akil H. 2000. Hormonal evidence for altered responsiveness to social stress in major depression. Neuropsychopharmacology 23: 411-418.

Received March 1, 2018; accepted in revised form September 11, 2018. 


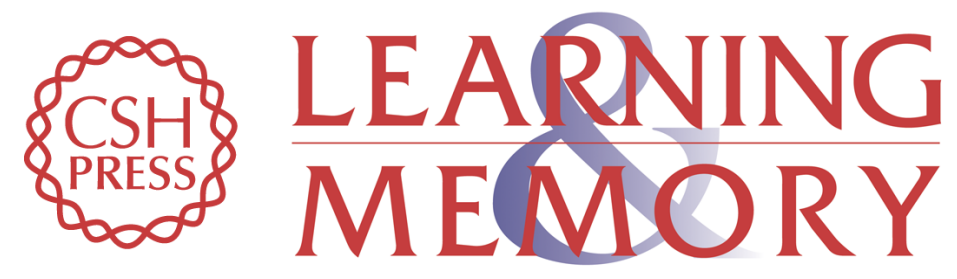

\section{Post-encoding stress enhances mnemonic discrimination of negative stimuli}

Tony J. Cunningham, Stephanie L. Leal, Michael A. Yassa, et al.

Learn. Mem. 2018, 25:

Access the most recent version at doi:10.1101/Im.047498.118

\begin{aligned} & \hline References $\begin{array}{l}\text { This article cites } 65 \text { articles, } 8 \text { of which can be accessed free at: } \\ \text { http://learnmem.cshlp.org/content/25/12/611.full.html\#ref-list-1 }\end{array} \\ & \begin{array}{r}\text { Creative } \\ \text { Commons } \\ \text { License }\end{array} \begin{array}{l}\text { This article is distributed exclusively by Cold Spring Harbor Laboratory Press for the } \\ \text { first } 12 \text { months after the full-issue publication date (see } \\ \text { http://learnmem.cshlp.org/site/misc/terms.xhtml). After } 12 \text { months, it is available under } \\ \text { a Creative Commons License (Attribution-NonCommercial } 4.0 \text { International), as } \\ \text { described at http://creativecommons.org/licenses/by-nc/4.0/. }\end{array} \\ & \begin{array}{c}\text { Receive free email alerts when new articles cite this article - sign up in the box at the } \\ \text { top right corner of the article or click here. }\end{array} \\ &$ Service \end{aligned}

